\title{
8-iso Prostaglandin F2alpha (8-iso-PGF2a)
}

National Cancer Institute

\section{Source}

National Cancer Institute. 8-iso Prostaglandin F2alpha (8-iso-PGF2a). NCI Thesaurus.

Code C92599.

A lipid peroxidation disease marker detected in the serum, biological fluids, and cell cultures using ELISA immunoassay kits. It is increased in patients with non-insulin dependent diabetes mellitus, coronary heart disease, and hypertension. 